\title{
The Spectrum Of Associated Congenital Anomalies in Disorders of Sex Development: A Review of the I-DSD Registry
}

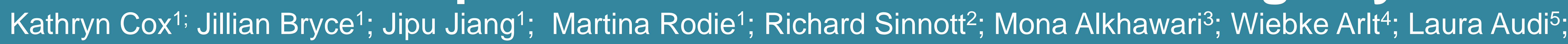
Antonio Balsamo6; Silvano Bertelloni7; Martine Cools ${ }^{8}$; Feyza Darendeliler9; Stenvert Drop ${ }^{10}$; Mona Ellaithi; ${ }^{11}$ Olaf Hiort $^{12}$; Paul-Martin Holterhus ${ }^{13}$; leuan Hughes ${ }^{14}$; Lidka Lisa ${ }^{15}$; Yves Morel'16; Olle Soder ${ }^{17}$; Peter Wieacker ${ }^{18}$; S. Faisal Ahmed ${ }^{1}$ University of Glasgow; ${ }^{2}$ University of Melbourne; ${ }^{3}$ Amiri Hospital, Kuwait; ${ }^{4}$ University of Birmingham; 5 Vall d'Hebron Research Institute, Barcelona; ${ }^{6}$ University of Bologna; 7 University Hospital Pisa; 8 University Hospital Ghent; 9 Istanbul University; ${ }^{10}$ Sophia Children's Hospital / Erasmus MC, Rotterdam; ${ }^{11}$ University of Khartoum; ${ }^{12}$ University of Lübeck; ${ }^{13}$ University Hospital of Schleswig Holstein, Kiel; ${ }^{14}$ University of Cambridge; ${ }^{15}$ Institute of Endocrinology, Prague; ${ }^{16}$ Hospices Civils de Lyon; 17Karolinska Institutet; ${ }^{18}$ Westfalian-Wilhelms University, Münster

\section{Introduction}

I-DSD is an international registry of cases with a Disorders of Sex Development (DSD). Information can be reviewed online in a nonidentifiable format by clinicians and researchers worldwide. This sharing of data facilitates research into the aetiology of these individually rare conditions, as well as their long-term outcome. The finding of associated anomalies in DSDs may indicate disruption of specific developmental pathways, targeting further research into causes. There is scarce information on the range of anomalies that may be associated with DSD.

\section{Aims}

To discover the frequency of specific associated anomalies in DSD, and to identify patterns of frequent anomalies within specific conditions.

\section{Methods}

Data were extracted from the I-DSD registry as at September 2012. At this point there were 1050 cases submitted by registered clinicians from 20 centres in 14 different countries. In 649 (62\%) cases there was a sufficient level of consent to allow sharing of suitable information. Case details were obtained from the Registry regarding diagnosis, genetic investigations and any associated congenital anomalies. Where information was unclear or incomplete the reporting clinician was contacted to obtain further information.

\section{Results}

Of 649 cases, congenital anomalies occurred in 173 (27\%); $107(62 \%)$ cases had one anomaly and 66 (38\%) had two or more anomalies. Associated anomalies are most frequently reported in Non-specific XY DSD (49\% of cases) and Disorders of gonadal development (41\%). (Figure 1). Within the cases with non-specific XY DSD 19\% of cases were reported to be small-for-gestationalage, $12 \%$ had cardiac abnormalities and $9 \%$ had conditions affecting the central nervous system.

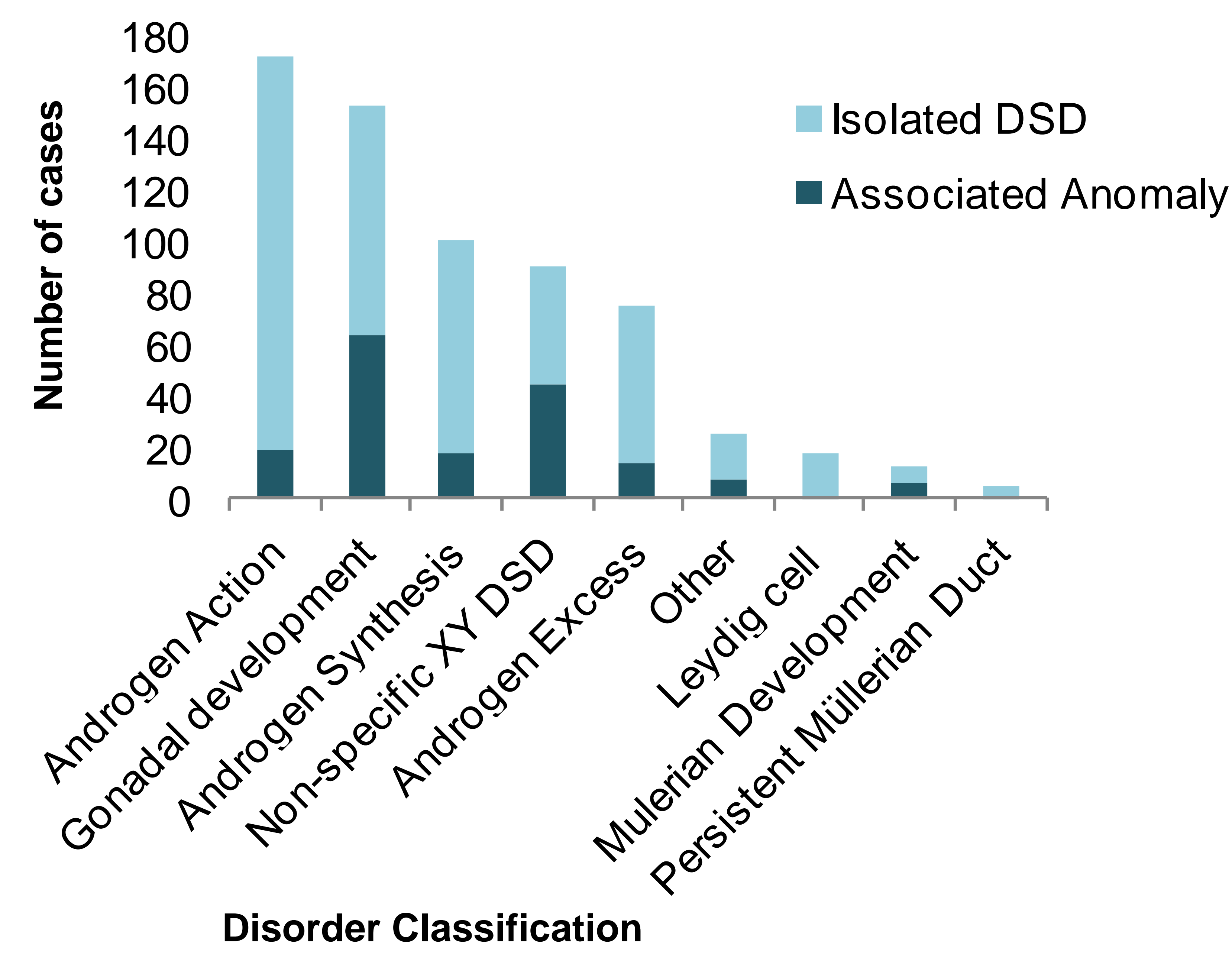

Figure 1: Relative frequency of anomalies according to disorder type

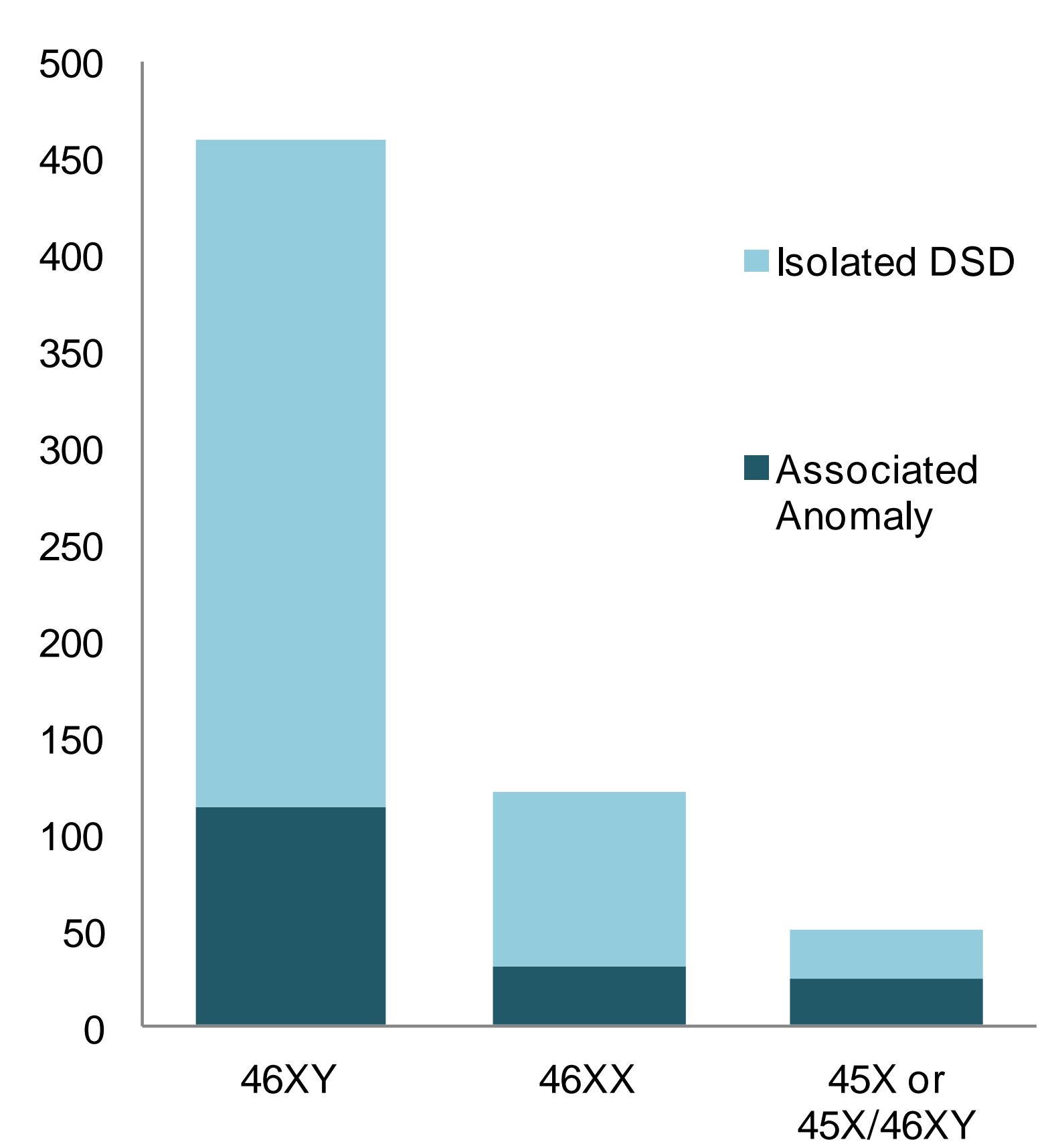

Figure 2: Frequency of anomalies according to karyotype

The majority of cases on the I-DSD registry are 46XY (71\%). Associated anomalies occurred with similar frequency in 46XY DSD(25\%) and 46XX DSD (26\%). (Figure 2). The range and distribution of associated anomalies differed substantially between cases of 46XY and 46XX DSD (Figure 3).

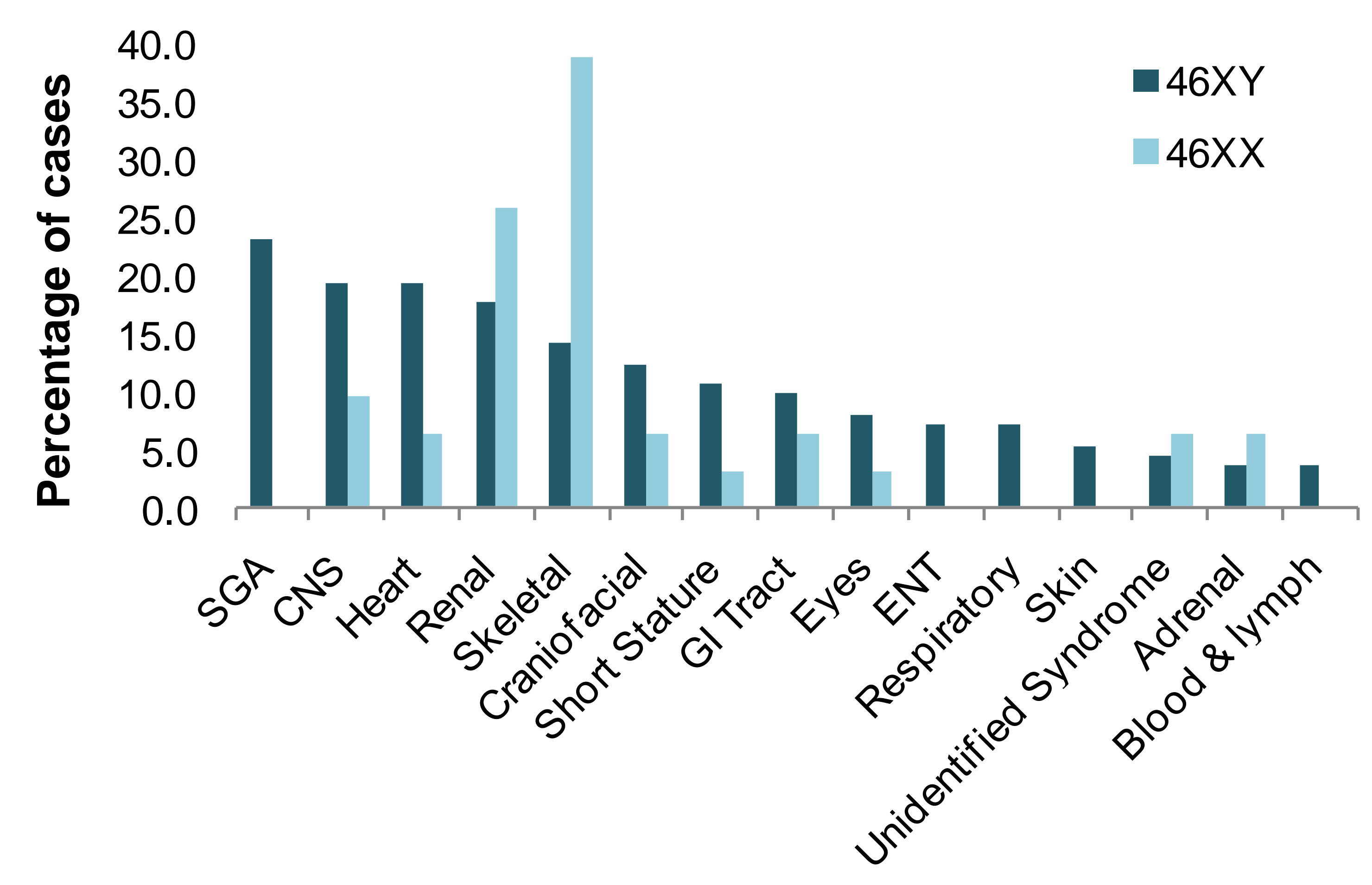

Figure 3: Range of anomalies differs between 46XY and 46XX DSD

Anomalies are also reported in conditions thought to involve isolated DSD such as androgen insensitivity syndrome. In the presence of confirmed androgen receptor mutations 10 cases had associated anomalies, most commonly skeletal (2) and renal (2). See Table 1.

Table 1: AR mutation status in cases with associated anomalies

\begin{tabular}{|lcc|} 
& CAIS & PAIS \\
\hline AR mutation present & 7 & 3 \\
\hline AR mutation absent & 1 & - \\
\hline AR mutation unknown & 1 & 7 \\
\hline Total & 9 & 10 \\
\hline
\end{tabular}

AR: Androgen Receptor, CAIS: Complete Androgen Insensitivity Syndrome, PAIS: Partial Androgen Insensitivity Syndrome

\section{Conclusion}

Associated anomalies are encountered frequently in a wide range of cases of DSD. These include monogenic conditions such as Androgen Insensitivity Syndrome which are generally thought solely to affect sex development. Anomalies are more frequently encountered in 46XY than 46XX DSD. As previously reported, SGA is frequent in 46XY DSD, but infrequent in 46XX DSD. In cases of 46XX skeletal and renal anomalies are most common. These findings provide a direction for further study of genetic and environmental causes of DSD. 